\title{
Development of francium atomic beam for the search of the electron electric dipole moment
}

Tomoya Sato ${ }^{1, a, b}$, S. Ando ${ }^{1}$, T. Aoki ${ }^{1}$, H. Arikawa ${ }^{1}$, S. Ezure ${ }^{1}$, K. Harada ${ }^{1}$, T. Hayamizu ${ }^{1}$, T. Inoue ${ }^{1}$, T. Ishikawa ${ }^{1}$, M. Itoh ${ }^{1}$, K. Kato ${ }^{1}$, T. Kato ${ }^{1, c}$ H. Kawamura ${ }^{1}$, H. S. Nataraj ${ }^{1, d}$, A. Uchiyama ${ }^{1}$, T. Aoki ${ }^{2}$, T. Furukawa ${ }^{3}$, A. Hatakeyama ${ }^{4}$, K. Hatanaka ${ }^{5}$, K. Imai ${ }^{6}$, T. Murakami ${ }^{7}$, Y. Shimizu ${ }^{8}$, T. Wakasa ${ }^{9}$, H. P. Yoshida ${ }^{5}$, and Y. Sakemi ${ }^{1}$

${ }^{1}$ Cyclotron and Radioisotope Center, Tohoku University, Miyagi 980-8578, Japan

${ }^{2}$ Graduate School of Arts and Sciences, University of Tokyo, Tokyo 153-8902, Japan

${ }^{3}$ Department of Physics, Tokyo Metropolitan University, Tokyo 192-0397, Japan

${ }^{4}$ Department of Applied Physics, Tokyo University of Agriculture and Technology, Tokyo 184-8588, Japan

${ }^{5}$ Research Center for Nuclear Physics, Osaka University, Osaka 567-0047, Japan

${ }^{6}$ Advanced Science Research Center, Japan Atomic Energy Agency, Ibaraki 319-1195, Japan

${ }^{7}$ Department of Physics, Kyoto University, Kyoto 606-8502, Japan

${ }^{8}$ Department of Physics, Tohoku University, Miyagi 980-8578, Japan

${ }^{9}$ Department of Physics, Kyushu University, Fukuoka 812-8581, Japan

\begin{abstract}
For the measurement of the electron electric dipole moment using Fr atoms, a Fr ion-atom conversion is one of the most critical process. An ion-atom converter based on the "orthotropic" type of Fr source has been developed. This converter is able to convert a few $\mathrm{keV} F r$ ion beam to a thermal atomic beam using a cycle of the surface ionization and neutralization. In this article, the development of the converter is reported.
\end{abstract}

\section{Introduction}

The existence of the permanent electric dipole moment (EDM) of elementary particles indicates a direct violation of time reversal symmetry. In a heavy atomic system, the bare value of the electron electric dipole moment (e-EDM) appears to be enhanced in an atomic EDM. In particular, in francium (Fr), the heaviest alkali atom, the enhancement is predicted to reach a factor of 895 [1]. Moreover, laser-cooling and trapping techniques proposed in the Cs system to improve the accuracy of the eEDM measurement greatly [2], is applicable for Fr.

For the search for the e-EDM using Fr, a factory to produce laser-cooled Fr atoms is being constructed at the Cyclotron and Radioisotope Center (CYRIC) at Tohoku University [3]. By the end of 2012, a Fr thermal ionizer had been developed, which is capable of producing $10^{6} \mathrm{Fr}$ ions/sec by a fusion reaction with ${ }^{18} \mathrm{O}$ primary beam and ${ }^{197} \mathrm{Au}$ target [4]. A beam line was constructed to deliver

\footnotetext{
ae-mail: sato@yap.nucl.ap.titech.ac.jp

${ }^{b}$ Present address: Department of Physics, Tokyo Institute of Technology, Tokyo 152-8551, Japan

${ }^{\mathrm{c}}$ Present address: Progress Technologies Inc.

${ }^{\mathrm{d}}$ Present address: Indian Institute of Technology Roorkee, Uttarakhand 247667, India
} 
the Fr ions 10 meters to a detector positioned safely away from the neutrons and $\gamma$ rays of the nuclear reaction and also apart from the electromagnetic fields generated by magnets in the primary beam line. In the next step, an efficient conversion of Fr ions to atoms and the transportation of Fr atoms to a magneto-optical trap (MOT) are the main objectives in achieving a precision e-EDM measurement.

\section{Development of an ion-atom converter}

In this experiment, Fr is provided as a few $\mathrm{keV}$ ion beam. The ion beam must be converted to a neutral atomic beam before proceeding with trapping the atoms and the EDM measurement. Therefore, a new type of ion-atom converter based on the "orthotropic" type of Fr source [5] has been developed.

On a hot metal surface, a thermal charge exchange process can occur between the metal and an ion (or atom). The ratio of the released ions to atoms depends on the work function $\left(E_{\mathrm{WF}}\right)$ of the metal surface and the ionization potential $\left(E_{\mathrm{IP}}\right)$ of the atom, according to Saha-Langmuir equation [6][7]. Under the condition of $E_{\mathrm{IP}}<E_{\mathrm{WF}}$, most of the atoms are released as ions. On the other hand, in the $E_{\mathrm{IP}}>E_{\mathrm{WF}}$ case, most of the ions are neutralized. In our converter, a combination of these processes plays an important role for producing a Fr atomic beam. Yttrium $\left(E_{\mathrm{WF}}=3.1 \mathrm{eV}\right)$ for neutralization and platinum $\left(E_{\mathrm{WF}}=5.6 \mathrm{eV}\right)$ for ionization were used to convert Fr whose $E_{\mathrm{IP}}$ is $4.0 \mathrm{eV}$.

The schematic view of the converter is shown in Fig. 1 (a). Fr ions enter through the injection hole and impact the oven wall of tantalum coated with platinum. The wall is maintained typically to approximately $1000^{\circ} \mathrm{C}$. Fr leaves the wall surface again as an ion but is now attracted to the yttrium target along a potential gradient generated by negative high voltage. From the surface of the target, Fr leaves as neutral atoms into $2 \pi$ steradians with velocities thermalized to a Boltzmann distribution in accord with the temperature of the target. The fraction of released atoms, some fraction that passes through a small hole at the oven wall is extracted as a thermal atomic beam [8]. The beam size and divergence are limited at the extraction hole. In this converter, the beam divergence is approximately $100 \mathrm{mrad}$. The other atoms that do not pass through the hole are captured by the platinum wall and continue the ionization-neutralization cycles until they pass through the hole. We discuss the efficiency of the converter in the next section.

The ion-atom converter minimizes the loss of Fr atoms by the recycling of the atoms that are emitted from the yttrium target at wider angles. After leaving the oven, the neutral beam is subjected to first transverse and then longitudinal cooling by what are standard laser-based practices applied to alkalis and other atoms [9] (Fig. 1 (b)). The transverse cooling is a kind of active collimation that ultimately reduces the transverse velocity of the atomic beam while preserving the total number of atoms [10]. In our case, the $33 \%$ of initial emittance of $100 \mathrm{mrad}$ will be reduced to $9 \mathrm{mrad}$. The longitudinal cooling is achieved by the Zeeman slower using magnetic fields and a laser light, which can slow down the atoms from a thermal energy to a low-temperature region appropriate to trapping in the MOT. Combining the converter with these apparatuses, the MOT will trap neutral Fr atoms with high efficiency. As the first step, the present report focuses on the yield of the Fr atomic beam. The collimation and slowing of the atomic beam will be the subjects of forthcoming reports.

\section{Fr neutralization}

The Fr neutralization experiment was performed using a Fr production beam line at CYRIC. A primary beam of ${ }^{18} \mathrm{O}^{5+}$ accelerated up to $100 \mathrm{MeV}$ by a $K=110 \mathrm{AVF}$ cyclotron was injected in the gold target installed in the Fr thermal ionizer, where Fr was produced through a fusion reaction ${ }^{18} \mathrm{O}+{ }^{197} \mathrm{Au} \rightarrow$ ${ }^{215-x} \mathrm{Fr}+x \mathrm{n}$. The produced Fr was extracted as an ion beam. It was transported in an approximately $10 \mathrm{~m}$ distance in the Fr transport beam line. Thereafter, ion beam was injected into the ion-atom 
(a)

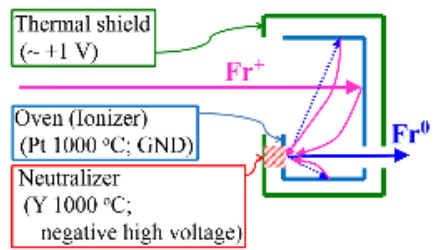

(b)

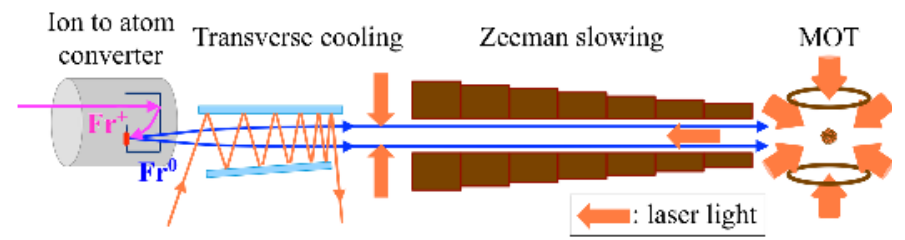

Figure 1. (a) Schematic view of the ion-atom converter. (b) Plan of the slow and collimated atomic beam production using transverse cooling and Zeeman slowing.

(a)

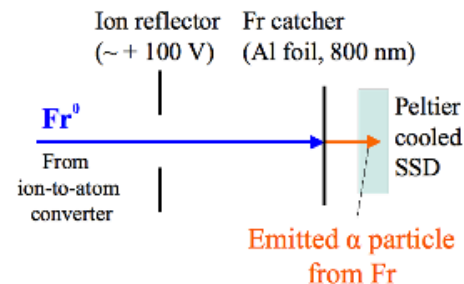

(b)

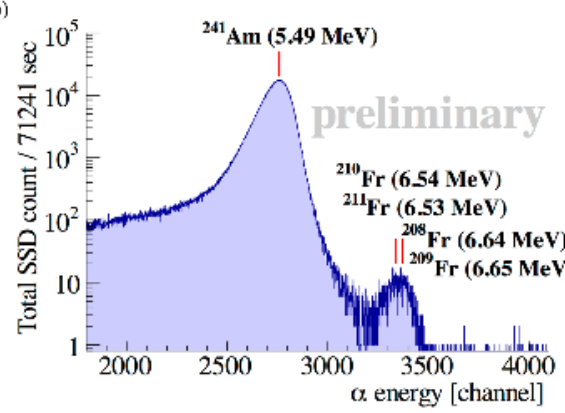

Figure 2. (a) Schematic view of the detection system. Only neutralized Fr atoms reach the catcher. Emitted $\alpha$ particles are detected by an SSD. (b) $\alpha$ energy spectrum measured with the SSD. The peak at $5.49 \mathrm{MeV}$ represents $\alpha$ particles from an ${ }^{241}$ Am energy calibration source.

converter. From the ion beam transport efficiency of the $\mathrm{Rb}$ ions, the Fr ion flux that injected to the converter was deduced as $2 \times 10^{3} \mathrm{pps}$. The output of the converter was caught by a detection system, which includes an ion reflector, a Fr catcher, and a Peltier-cooled silicon solid-state detector (SSD), as shown in Fig. 2 (a). Because an SSD is vulnerable to a light and heating, they would cause a deterioration of the energy resolution and damage the SSD itself. To avoid damages by direct radiation from the hot converter, the Fr catcher was inserted between the converter and the SSD. After Fr was attached to this catcher, the $\alpha$ particle emitted from Fr was detected by the SSD. The Peltier device was water cooled and kept the SSD at approximately $12^{\circ} \mathrm{C}$ despite the operation of the converter at temperatures of $\geq 1000^{\circ} \mathrm{C}$. The temperature was measured by thermocouples.

The energy spectrum of $\alpha$ particles detected by the SSD was shown in Fig. 2 (b). The signals occurred only on delivery of the Fr ion beam. Also, it was concluded that the peak at $6.6 \mathrm{MeV}$ comprised $\alpha$ particles from the decays of ${ }^{210} \mathrm{Fr},{ }^{211} \mathrm{Fr},{ }^{208} \mathrm{Fr}$ and ${ }^{209} \mathrm{Fr}$. The energy resolution was not sufficient to separate the $\alpha$ energy of each isotope. However, on considering the activities of the isotopes and the branching ratios of the fusion reaction, we estimate that the peak was dominated by the $\alpha$ from ${ }^{210} \mathrm{Fr}$. The Fr neutralization efficiency of the converter was approximately $0.06 \%$.

Figure 3 (a) shows the dependence of the SSD count rate on the converter temperature. The SSD count rate increased as the temperature of the converter rose. This trend is attributed to activation of the Fr release process from the inner surface of the converter. Figure 3 (b) shows the dependence of the SSD count rate on neutralizer voltage. The count rate at the SSD increased as the negative voltage of the neutralizer rose. This trend could result from the increase of the attracted ions to the neutralization target. The SSD count rate might not have reached the maximum within the ranges of temperature and voltage covered here because of the heat resistance property of the thermal shields and leakage current of the high voltage supply. 

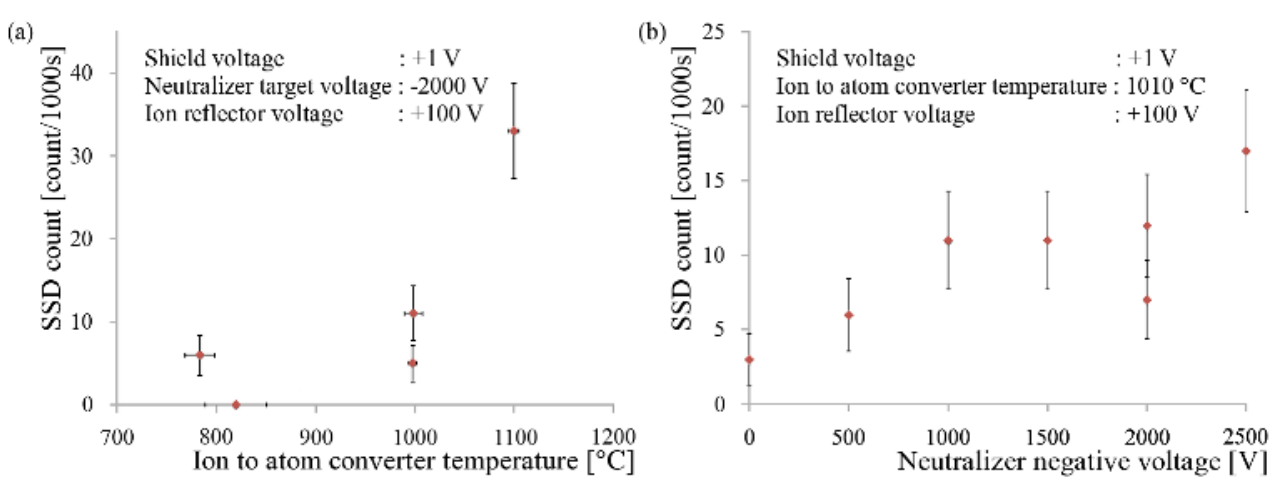

Figure 3. Dependence of the SSD count rate on (a) the temperature of the ion-atom converter, and (b) the voltage for the yttrium neutralizer target.

\section{Summary and Future plan}

We have developed a new Fr ion-atom converter based on the "orthotropic" Fr source. It is able to make a thermal Fr atomic beam from a few $\mathrm{keV}$ Fr ion beam using a cycle of surface ionization and neutralization. In the on-line experiment, we have confirmed the neutralization of Fr ions ejected from the Fr thermal ionizer. Increase of the ion-atom conversion efficiency is necessary for our experimental plan. To improve the e-EDM limit, at least $10 \%$ of the injected Fr ions must be extracted as atoms. The efficiency in the present status is limited by unexpected dropouts of Fr. Reducing the size of the ion injection hole and redesign of the oven shape should reduce the loss of Fr. Elevation of the temperature and voltage seems to be effective in increasing the efficiency. The maximum possible conversion efficiency will be $10 \%$ including the decay of Fr in the converter. Obviously, an extended study of Fr release and absorption processes at the converter wall would be valuable. To enhance the quality of the atomic beam, designing of the transverse cooling and Zeeman slower is in progress.

The authors appreciate the staffs of CYRIC machine group for their cooperation during experiments. Comments given by Prof. K. Asahi and Dr. Y. Ichikawa have been a great help in writing the paper. T.S. and T.H. would like to thank the JSPS Research Fellowships for Young Scientists. This work was mostly supported by Grants-in-Aid for Scientific Research (Nos. 21104005 and 23740166) and Tohoku University's Focused Research Project.

\section{References}

[1] D. Mukherjee, B. K. Sahoo, H. S. Nataraj and B. P. Das, J. Phys. Chem. A 113, 12549 (2009)

[2] C. Chin et al., Phys. Rev. A 63, 033401 (2001)

[3] Y. Sakemi et al., J. Phys.: Conf. Ser. 302, 012051 (2011)

[4] H. Yoshida et al., CYRIC Annual Report 2010-2011, 4 (2012)

[5] T. Dinneen, A. Ghiorso and H. Gould, Rev. Sci. Instrum. 67, 752 (1996)

[6] I. Langmuir and K. H. Kingdon, Proc. Roy. Soc. A 107, 61 (1925)

[7] M. J. Dresser, J. Appl. Phys. 39, 338 (1968)

[8] N. Ramsey, Molecular Beams (Oxford University Press, Oxford, 1986), 19

[9] H. J. Metcalf and P. Van der Straten, Laser Cooling and Trapping (Springer, Berlin, 1999)

[10] C. Slowe, L. Vernac and L.V. Hau, Rev. Sci. Instrum. 76, 103101 (2005) 